\title{
REVIEW
}

\section{Peripheral Nerve Grafts Support Regeneration after Spinal Cord Injury}

\author{
Marie-Pascale Côté, Arthi A. Amin, Veronica J. Tom and John D. Houle \\ Department of Neurobiology and Anatomy, Spinal Cord Research Center, 2900 Queen Lane, Drexel University College \\ of Medicine, Philadelphia, Pennsylvania 19129
}

\begin{abstract}
Summary: Traumatic insults to the spinal cord induce both immediate mechanical damage and subsequent tissue degeneration leading to a substantial physiological, biochemical, and functional reorganization of the spinal cord. Various spinal cord injury (SCI) models have shown the adaptive potential of the spinal cord and its limitations in the case of total or partial absence of supraspinal influence. Meaningful recovery of function after SCI will most likely result from a combination of therapeutic strategies, including neural tissue transplants, exogenous neurotrophic factors, elimination of inhibitory molecules, functional sensorimotor training, and/or electrical stimulation of paralyzed muscles or spinal circuits. Peripheral nerve grafts provide a growthpermissive substratum and local neurotrophic factors to enhance the regenerative effort of axotomized neurons when
\end{abstract}

grafted into the site of injury. Regenerating axons can be directed via the peripheral nerve graft toward an appropriate target, but they fail to extend beyond the distal graft-host interface because of the deposition of growth inhibitors at the site of SCI. One method to facilitate the emergence of axons from a graft into the spinal cord is to digest the chondroitin sulfate proteoglycans that are associated with a glial scar. Importantly, regenerating axons that do exit the graft are capable of forming functional synaptic contacts. These results have been demonstrated in acute injury models in rats and cats and after a chronic injury in rats and have important implications for our continuing efforts to promote structural and functional repair after SCI. Key Words: Spinal cord injury, peripheral nerve graft, axon regeneration, transplantation, neuroplasticity, chondroitinase.

\section{INTRODUCTION}

Significant strides have been made in our understanding of the potential for regeneration by injured adult mammalian central nervous system (CNS) neurons. It has been long known that while axons in the peripheral nervous system (PNS) are able to regenerate after being severed, this does not hold true for injured CNS axons. The reason for this dichotomy was unclear until the landmark studies of Bray, Aguayo, David, and Richardson et al. [1-6] in the early 1980s suggested that the environmental milieu available to injured PNS axons might be more favorable than that encountered by injured CNS axons. David and Aguayo [3] elegantly demonstrated that when injured CNS axons are provided with a supportive

Electronic supplementary material The online version of this article (doi:10.1007/s13311-011-0024-6) contains supplementary material, which is available to authorized users.

Address correspondence and reprint requests to: John D. Houle, Ph.D., Department of Neurobiology and Anatomy, Spinal Cord Research Center, Drexel University College of Medicine, Philadelphia, PA 19129, USA. E-mail: jhoule@drexelmed.edu. substratum, such as a segment of peripheral nerve, they are capable of regenerating for long distances and of being directed toward a specific targeted area. Since then, many investigators have built onto this seminal finding and have established using peripheral nerve grafts (PNGs) as a viable therapeutic treatment of the injured spinal cord [7-10]. Here, we will discuss some basic technical aspects of using a PNG to promote regeneration, the application of PNGs to a variety of injury conditions (including a delayed grafting [chronic injury] approach), the use of PNGs in a combinatorial treatment strategy, and the translation of peripheral nerve (PN) grafting to large animal models of spinal cord injury (SCI). This review focuses on results obtained from grafting peripheral nerve segments into the injured spinal cord, but it would be remiss not to mention that alternative approaches using components of peripheral nerves (e.g., purified Schwann cells in implantable guidance channels [11-21], or the re-routing of a peripheral nerve from its target muscle to enter the spinal cord distal to an injury [2224]) have demonstrated considerable success in establishing connectivity across a lesion site. Notably, the number of 
autologous Schwann cells can be expanded in culture prior to transplantation so that large lesion areas can be completely filled. Additionally, there is the potential to transfect these cells to produce growth-promoting factors before transplantation. In our experience, PNGs have the benefit of Schwann cells being aligned in a linear array at the time of transplantation, which seems to facilitate early axon ingrowth. PNGs also are a flexible cable that can be situated extradurally for easy electrophysiological testing.

\section{Technical considerations for using a peripheral nerve graft}

The pioneering work of Aguayo and colleagues established many of the basic principles by which a PNG could be used to measure the potential for regeneration by CNS neurons. Their initial studies indicated that neurons close to the insertion site were most likely to be involved in the regenerative response. That is, a graft at a cervical spinal cord level would contain axons from cervical propriospinal neurons and from brain stem neurons, while a graft at thoracic or lumbar levels would contain axons from local propriospinal neurons but not brain stem neurons [6]. This observation helped establish the belief that distance of the lesion/graft from the neuron cell body was critical to whether a regenerative response was initiated by placement of the PNG. Early studies also established that only axons that had been injured appeared to grow into the graft and not collateral sprouts from non-injured, adjacent axons [25]. This observation indicated that a PNG serves as a structure where the properties of an isolated population of regenerating axons may be studied. This has several advantages over other neural tissue transplantation procedures. The central portion of a PN bridge can be physically isolated from surrounding tissues, making it easily accessible for tract tracing studies, for direct electrical stimulation to test conduction by regenerating axons without current spread into the spinal cord and for the testing of the specific impact of regenerating axons on behavioral recovery since the graft can be sectioned without causing damage to adjacent spinal cord tissue. In this situation, loss of a previously recovered function due to the cutting of a PNG would be strong evidence that axons in the graft had contributed significantly to the recovery [26].

It was determined that pre-degenerated (i.e., segments of nerve cut and left in situ for 7-10 days prior to harvesting) peripheral nerves are infiltrated by regenerating axons to a greater extent (both in number and distance) than when freshly cut and harvested nerves are used $[27,28]$. This suggested that the local non-neuronal cell response to injury during the process of axon degeneration might be involved in encouraging CNS axons to regenerate into the $\mathrm{PNG}$, similar to what was observed with PNS axon regeneration. Further study confirmed that extensive structural reorganization of degenerating myelinated axons into hollow tubes with a laminin layer (Bands of Büngner) act as physical guides for linear, long distance growth of axons through the graft $[29,30]$. Also it was suggested that Schwann cells were activated by PN injury resulting in an upregulation of neurotrophic factor production that may be important for attracting axonal ingrowth and supporting long distance growth through the graft [31, 32]. Messenger RNA data indicates that levels of NGF and GDNF, but not BDNF or NT3, are elevated in PNGs at 10 days and 20 days after grafting when comparing levels in the PNG to adjacent spinal cord tissue [33]. Acellular grafts (resulting from a freeze-thaw procedure to kill Schwann cells and fibroblasts) were found to be far less effective in supporting axonal growth, despite the presence of laminin coated Bands of Büngner [9, 34, 35]. Another advantage with using a PNG over other neural tissues for transplantation is that Schwann cells enthusiastically form myelin sheaths around many/most of the axons within the PNG, bestowing on these regenerated fibers the ability to efficiently conduct action potentials at or near rates observed for intact spinal cord axons [36, 37].

There does not appear to be a restriction on the length to which regenerating axons will grow, as the majority of axons extend to the distal end of a PNG irrespective of its length [1]. We have evidence of axons growing over $4 \mathrm{~cm}$ within grafts inserted into the injured cat spinal cord (for more detail, see Côté et al. [36]) and there is no reason to believe that growth in longer grafts would not be possible. It is important to have this flexibility with regard to the length of grafts so that regenerating axons at the distal end can be apposed to a specific target area (such as a particular level of spinal cord, either a dorsal or ventral region, or gray matter vs. white matter). Axons reaching the distal end are presented with the opportunity to extend back into the spinal cord, yet the majority remain within the graft. This is true no matter what the source of axons within the graft or the region of apposition, and this represents the next major hurdle to be overcome in the field of transplantation and regeneration. There are a few axons that will cross the graft-host interface and extend for short distances $(<100 \mu \mathrm{m})$ without any manipulation of the interface, but significantly more axons $(10-15 \%$ of those that have grown into the graft) will exit the graft and extend for longer distances $(>500 \mu \mathrm{m})$ after modulation of the inhibitory extracellular matrix by application of the bacterial enzyme chondroitinase (ChABC) [26, 38]. This combination of treatment strategies to enhance regeneration beyond the PNG will be discussed in more detail below.

From a practical standpoint, the source of nerve for grafting does not appear to matter when the extent of axonal ingrowth in considered. A typical experiment in many laboratories will utilize the sciatic nerve or one of 
its branches (tibial or peroneal nerve) because of their large size relative to the spinal cord injury site. In the case of a complete transection lesion 2 pieces of tibial nerve placed into the cavity side by side are sufficient to appose most of the surface area of the injured spinal cord. Others have used multiple pieces of smaller intercostal nerves stabilized by fibrin glue to fill a complete transection lesion [39-41] or multiple nerve segments embedded in a pre-formed matrix [42]. In these latter situations both ends of the grafts simultaneously were apposed to the injured spinal cord, fitting between the cut ends of the complete spinal cord lesion site, allowing for bidirectional growth. This approach is most efficient when there is a short lesion cavity to be "spanned". In contrast is the situation where one end of the PNG is apposed first, to allow axons to enter the graft and grow to the distal end before being apposed to the spinal cord. The length or size of spinal cord injury does not impact on the use of this "bridging" approach. The pros and cons for each approach will be discussed in more detail below, but one reason for delaying the grafting of one end is that this restricts the type of axons that eventually fill the graft to being primarily descending (motor) or ascending (sensory) axons, allowing the investigator to focus on one functional type or the other. It also makes it possible to have 2 grafts in the same animal, 1 for descending and 1 for ascending axons, to study the response by both fiber types to different treatments (see below).

A final consideration is that there is a readily available source of peripheral nerves within the subject that can be used as autologous grafts. This approach obviates the need for long term immunosuppression to prevent graft rejection and substantiates the potential of applying a peripheral nerve grafting approach to a clinical setting. While some loss of function likely will result from harvesting of autologous nerves, it is possible to use a purely cutaneous nerve (such as the sural nerve) to minimize further loss of motor function in the SCI subject.

\section{Experimental approaches using a PNG}

A video format of the complete surgical procedure has been published in the Journal of Visualized Experiments (JoVE) [43] so that investigators can explore this treatment strategy for incorporation into their own experimentation.

In the simplest situation involving a small, acute injury, a pre-degenerated segment of peripheral nerve can be harvested and immediately placed into the spinal cord, spanning the cuts ends of the cord (FIG. 1a). The extent of injury in terms of region of spinal cord affected does not impact on the success of this approach, as a partial hemisection lesion up to a complete transection lesion has been treated in this manner. An acute contusion injury is difficult to treat with this approach because the cavity necessary to accommodate the PNG does not form for at least 7 days (in rats) after injury. Even then, there is a need to remove cellular debris from the lesion epicenter for correct placement of the graft. When dealing with a sub-chronic or a chronic ( $>4$ weeks) injury, a spatial and temporal sequence of surgical procedures and treatment strategies (FIG. 1b) must be instituted to facilitate axonal growth into and beyond the graft back into the spinal cord.

The isolation and cutting of the target peripheral nerve is the first step for any of the treatment approaches using a PNG. Routinely we use the tibial nerve in the hind limb (autologous or heterologous), making a ligation and cut at the branch point from the sciatic nerve, at a mid-thigh level. The cut nerve is left in situ and overlying muscle and skin closed. After 7-10 days the distal nerve segment is harvested just prior to being grafted into the injured spinal cord. For an acute spanning procedure the perineurium is stripped from both ends of the nerve segment to decrease the formation of scar tissue by fibroblasts that might infiltrate into the graft-host interface. The length of the nerve segment is trimmed to fit snuggly against the cut ends of the lesion and inserted lengthwise into the cavity, sometimes with fibrin glue added to secure the graft ends to spinal cord tissue [39]. The dura mater is sutured over the PNG to keep it in place. For an acute bridging nerve graft the perineurium is stripped from the end of the nerve segment to be apposed to one end of the lesion cavity. The PNG is secured in place by suturing the perineurium to the dura mater followed by a partial closure of the dura mater over the proximal end of the PNG. The distal end of the PNG is left unapposed to the spinal cord, with the length of the nerve segment lying on top of the dura mater. Apposition of the distal end is delayed to allow time for axons to regenerate into and through the graft, so that they are in an active growth mode when the distal end eventually is apposed. Regeneration into the PNG begins around 710 days after grafting and axons reach the distal end 23 weeks later. For longer grafts, as in the cat, a longer period (5-6 weeks) for axon extension through the graft is necessary. The next procedure is to create a fresh lesion for apposition of the distal end of the PNG. Usually this entails an aspiration lesion of the dorsal quadrant of the spinal cord several segments from the proximal apposition site. Upon hemostasis the distal end of the PNG is trimmed by $1-2 \mathrm{~mm}$, perineurium is stripped back and the distal end is inserted firmly into the cavity and secured by suturing perineurium to dura mater. The strategy for grafting into a chronic injury requires that scar tissue be surgically removed by micro scissors and aspiration before the proximal end of the nerve segment can be apposed. Microinjections of ChABC and the neurotrophic factor GDNF are made 
a

PNG - span

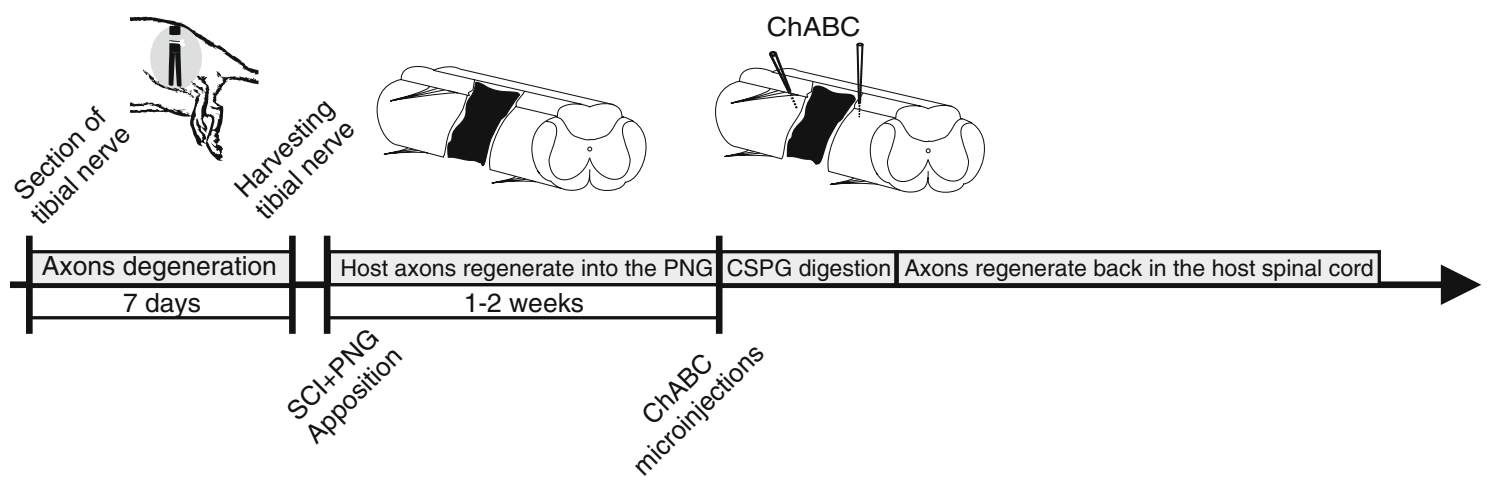

b

\section{PNG - bridge}

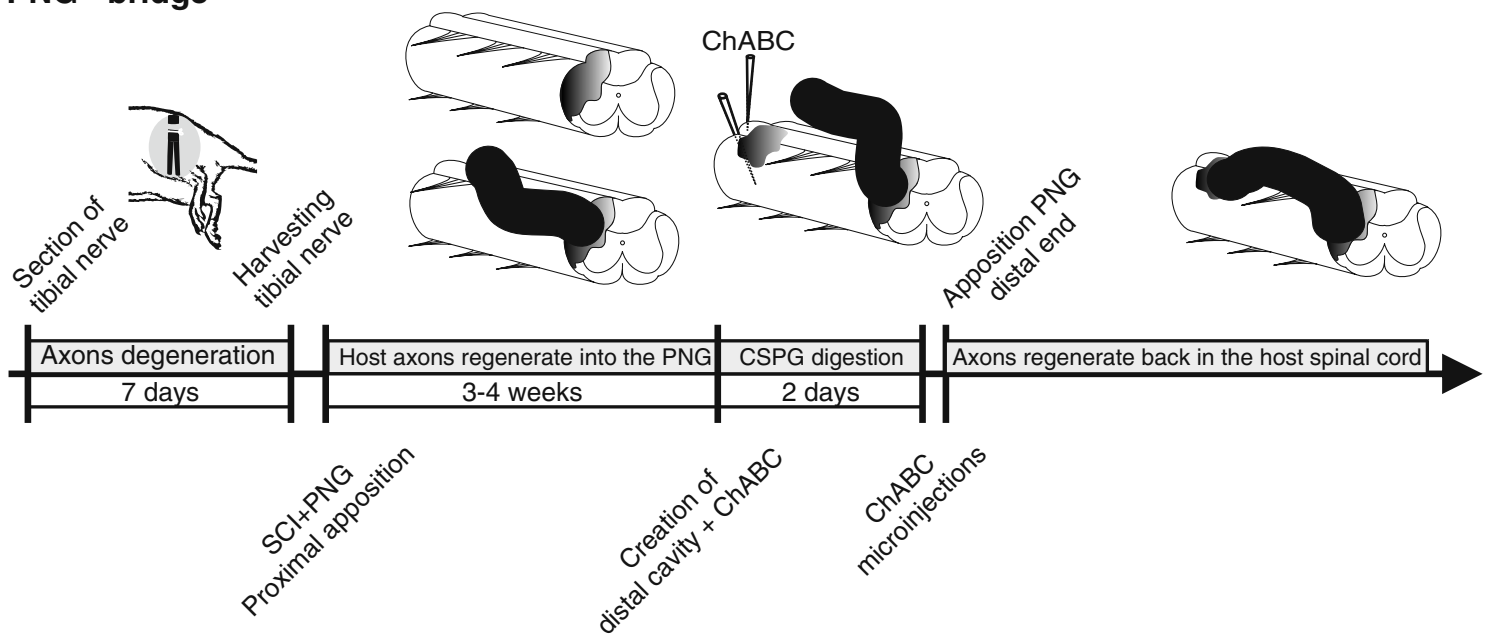

FIG. 1. Surgical procedures and timeline for the implantation of PNGs that span (a) or bridge (b) a spinal cord lesion. ChABC bacterial enzyme chondroitinase; CSPG cavity to digest proteoglycan; PNG peripheral nerve graft; $S C l$ spinal cord injury.

into and several $\mathrm{mm}$ rostral to the cavity to digest proteoglycans (CSPGs) that are inhibitory to axonal growth and to activate a regenerative program within chronically injured neurons [26, 44, 45], respectively. Work with chronically injured rats has shown that this combination treatment is necessary to achieve axonal ingrowth into the proximal end of the PNG that is comparable to an acutely placed graft [46]. The proximal end of the graft is apposed and secured as with the acute grafts and the distal end left unapposed, extradurally above the spinal cord. After allowing sufficient time for axons to grow to the distal end the procedure for apposing the distal end is as described for the acute grafts except that $\mathrm{ChABC}$ is microinjected into and around the fresh lesion site and then again 3 days later, before the distal end is apposed.

There are several ways that axons within and beyond the graft can be examined. Anatomically, anterograde tracers, such as biotinylated dextran amine (BDA), can be microinjected into specific regions of the brain stem or spinal cord to label descending spinal pathways that contribute axons to the PNG. In this case, a survival time of 1-2 weeks is necessary to allow complete transport of the tracer into distal axons to label all axons within the graft but without direct identification of their source.

The determination of successful regeneration requires electrophysiological measures to establish conduction along regrowing axons and the formation of functional synapses. We have indication of functional regeneration in a complete transection rat model with a PNG that spans the lesion site (FIG. 2a). Cord dorsum potentials were recorded from the surface of the spinal cord below the lesion site following intraspinal stimulation of the cord rostral to the graft. This illustrates that electrical activity can be transmitted by regenerated axons in the graft since lidocaine injection caused a sharp decrease of the amplitude and an increase in response threshold. Motor-evoked potentials are also frequently used to assess conduction in PNGs after a complete SCI. We also have observed recovery of magnetically motorevoked potentials 6 weeks after a complete transection and PNG (FIG. 2b). Anatomical evidence of synaptic 
a

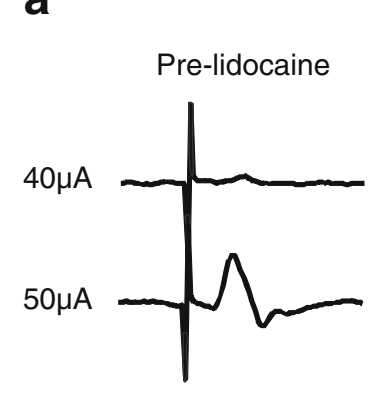

$100 \mu \mathrm{A}$

$200 \mu \mathrm{A}$

$400 \mu \mathrm{A}$

\section{Evoked potentials}

$8 \mathrm{~min}$ post-lidocaine $8 \mathrm{~min}$ post-lidocaine

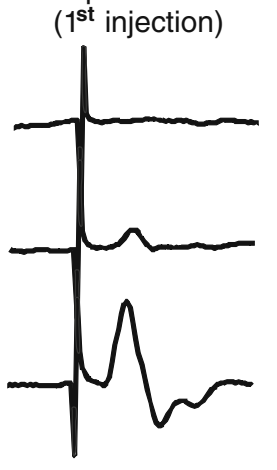

( $2^{\text {nd }}$ injection)

Cord dorsum potential

EMG - adjacent back muscle

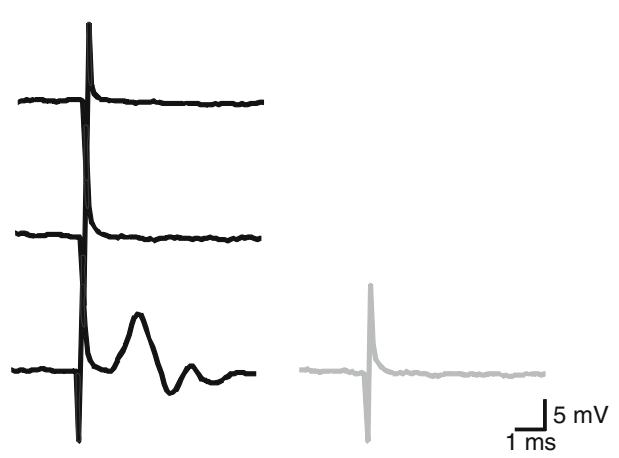

b

\section{Magnetic motor evoked potentials}

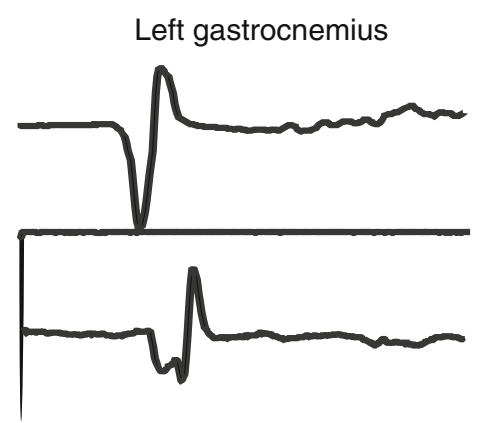

Right gastrocnemius

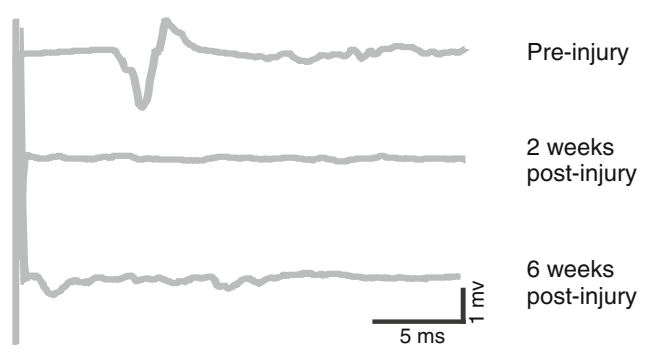

FIG. 2. a, Cord dorsum potentials recorded from the spinal cord and axial musculature surrounding the cord before and after 2 successive injections of lidocaine in the PNG in response to the stimulation of the spinal cord rostral to the PNG. Interelectrode distance: $\sim 6 \mathrm{~mm}$. b. Magnetic motor-evoked potentials recorded from left and right gastrocnemius muscle before and after a complete thoracic $\mathrm{SCl}$ and spanning PNG. EMG=electromyogram.

activity between regenerated axons in the PNG and spinal cord neurons is established by an increase in the number of cFos immunoreactive neurons near the graft-host interface after electrical stimulation of the PNG [38, 47].

\section{Regeneration after acute SCI}

Work in the mid to late 1980's advanced our understanding of the variety of responses by axotomized neurons to the presence of a PNG [48] and the first demonstration of a return of physiological activity by the connection of a PNG between medullary respiratory neurons and neurons in the phrenic nucleus [49]. Cheng and Olsen [39] described the use of multiple intercostal nerves to span a complete transection lesion, in combination with fibrin glue and $\alpha \mathrm{FGF}$. These PNGs were apposed such that white matter motor tracts aligned with grey matter motor neuron pools and ascending white matter tracts with grey matter sensory neuron pools. This transplantation study has resulted in behavioral, electrophysiological, and anatomical improvements. Forelimb stepping, partial weight support, and movement in all three joints were observed after PNG $+\alpha$ FGF treatment $[39,40]$. Motor-evoked potentials [41, 42] and somatosensory potentials [40] were recorded from animals that received this transplantation strategy. Serotonin positive fibers found in the cord caudal to the PNG indicated axonal outgrowth from the graft [41]. Results from standardizing the connection of intercostals nerves from grey matter to white matter by a prosthesis device show that the device remains correctly positioned six months after grafting and recording of motor-evoked potentials can be obtained from $80 \%$ of the animals [42]. 


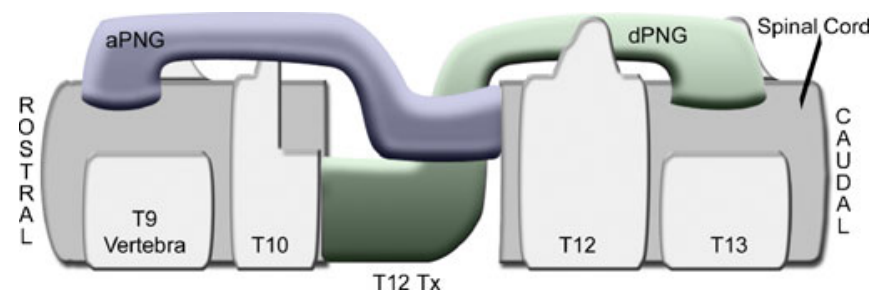

FIG. 3. Schematic illustration of sagittal view shows sciatic branches grafted into a lower complete thoracic injury. Tibial branch is apposed to the rostral wall and peroneal branch is apposed to the caudal wall. aPNG peripheral nerve graft with ascending axons; $d P N G$ peripheral nerve graft with descending axons.

Descending regenerating axons cannot be distinguished from ascending regenerating axons within these spanning grafts, but this can be overcome by apposing individual branches of the sciatic nerve as bridging grafts to either rostral or caudal wall of the lesion (FIG. 3). Delaying distal end apposition of each PNG allows the separation of descending regeneration in the rostrally apposed PNG from ascending regeneration in the caudally apposed PNG. Currently we are examining the effects of exerciseinduced increase in neurotrophic factors in the spinal cord above and below the level of injury [50] to determine if a local increase in endogenous factors might improve axonal regeneration into, and possibly out of, these differently apposed PNGs.

Our first study of regeneration into a PNG in rats was completed almost 20 years ago [51] when we demonstrated that propriospinal neurons were retrogradely labeled with True Blue that had been applied to the distal end of a graft that had been apposed to a upper lumbar level spinal cord injury site. Since then we have demonstrated that neurotrophic factors are necessary to encourage regeneration by chronically injured neurons [46]; that growth beyond the distal end of the graft is enhanced by treating the area with $\mathrm{ChABC}$ leading to some return of functional activity [26]; that PNGs can be placed into a cervical contusion injury without causing secondary behavioral deficits and still support regeneration [52]; that the combination of ChABC and PNG in a chronic contusion injury model will lead to axonal regeneration, formation of functional synapses across the lesion and some degree of behavioral recovery [38]. Currently we are testing whether forced exercise of the impaired fore limb [52] will lead to improved functional recovery in chronically injured rats that receive a PNG and ChABC treatment. Together these studies establish the general approach of PN grafting experiments and provide insight into the unique features of grafting after a chronic injury that must be dealt with for clinical success.

Another set of experiments involves a combined treatment approach where $\mathrm{ChABC}$ was provided at the rostral PNG-spinal cord interface to encourage axonal growth into the graft and at the distal PNG-spinal cord interface to encourage axonal outgrowth back into the spinal cord. Retrograde tracing experiments demonstra- ted that the majority of axons growing into a lower thoracic level graft are from thoracic propriospinal neurons, with some contribution by reticulospinal and vestibulospinal neurons [33]. Extension of regenerated axons beyond the distal PNG-spinal cord interface was observed by anterograde tracing experiments and electrical stimulation of the PNG promoted cFos expression in distal spinal neurons, which was indicative of synaptic activity by regenerated axons exiting the graft. Currently we are performing this grafting approach in combination with hind limb training (either bicycling or stepping) for rats [50], to assess changes in motoneuron excitability after SCI and the possibility of attracting regenerating axons towards the CPG of the lumbar spinal cord in response to the rhythmic activity during training.

\section{Regeneration by chronically injured axons}

The vast majority of SCI patients are living with injuries they sustained long ago. Chronically injured neurons exist in a long-term atrophic state and have lowlevel expression of regeneration-associated genes (RAGs) [53, 54] suggesting that long-injured axons have an even lower propensity to regrow than do freshly injured axons. Complicating the issue is the observation that the glial scar tissue surrounding the injury site is significantly denser than what is present after an acute lesion. Several groups, including ours, have physically removed (debrided) and/or chemically modulated the established scar tissue to maximize axon penetration into a growth-promoting substratum, such as a PNG [51, 55]. Debridement may also serve to promote outgrowth by relesioning long-injured axons. Surprisingly, injuring axons a second time does not lead to additional cell loss [56] but instead initiates a rapid increase in expression of BII-tubulin and growth-associated protein-43 [53]. Debridement is not the only method that is able to spark a regenerative response of chronically injured axons. Treatment of a chronic lesion cavity with neurotrophic factors, such as BDNF or GDNF, prior to transplantation of a PN results in increased expression of RAGs [45] and pronounced regeneration of long-injured axons into the PNG [46]. Moreover, robust regrowth of chronically injured axons into a PN is seen following delayed GDNF treatment of a one or two month-old lesion cavity in 
which scar tissue was left intact [38, 52]. Administering neurotrophic factors to the cell bodies of long-injured axons also appears to have positive effects. Kwon and colleagues [57] delivered BDNF into the red nucleus one year after injuring the rubrospinal tract and found that BDNF-treatment reversed cell atrophy, increased RAG expression, and promoted axonal regeneration.

Multiple groups have demonstrated that long-injured supraspinal axons are able to extend into various types of delayed grafts [58-60]. As with acute SCI, regeneration of chronically injured axons into a PNG overcomes but one hurdle; getting axons to extend out of the graft to reform synapses distal to the injury is, perhaps, an even larger stumbling block. We hypothesized that combining a PNG bridge and ChABC, similar to our acute injury strategy [26], would also be effective in a chronic injury setting. Indeed, we found that chronically injured axons that grew into the PNG emerged from it and reinnervated the distal spinal cord [38], leading to behavioral improvements and expression of cFos in neurons beyond the distal interface when the PNG was electrically stimulated, indicating that axons within the graft formed active synapses.

Although it is very encouraging to observe functional regeneration by chronically injured axons, it is important to note that thousands of axons remain "trapped" within the distal PNG and it is our challenge to develop new strategies to "encourage" greater outgrowth. Simply increasing BDNF levels distal the graft did not further enhance chronically axons' ability to regenerate out of the graft as similar numbers of axons were found following ChABC-treatment or ChABC+BDNF-treatment, but it did increase the number of cFos + neurons distal to the graft compared to just BDNF or just ChABC treatment. Thus, there appears to be a synergism between ChABC-mediated promotion of axonal regeneration and BDNF expression in the distal spinal cord that may either enhance the ability of regenerated axons to form synapses or improve synaptic efficiency. Future considerations for strategies to "push" axons beyond the distal interface to reinnervate spinal cord include those that have been shown to improve the intrinsic ability of mature axons to regrow; such as manipulating genes associated with protein synthesis (PTEN/mTOR) [61], administering neurotrophins to the cell body in addition to the lesion cavity [57] or electrical stimulation of neurons or their regrowing axons [62,63].

\section{Functional recovery with PNGs}

Despite several reports of successful axonal growth within a PNG, there are few studies that correlate evidence of anatomical repair with electrophysiological or functional recovery. This disparity is most likely due to the poor re-entry of regenerated axons back into the host spinal cord. Conversely, some groups have shown evidence of functional recovery after a PNG inserted into a complete lesion cavity but this recovery, in many cases, is not supported by anatomical evidence of axonal growth. For example, motor-evoked potentials with a delayed latency 6 months after a complete thoracic transection and implantation of a molded prosthesis containing 12 peripheral nerve grafts (intercostal nerves) were observed but the axons that grew into the grafts did not exit the transition zone between the graft and spinal cord [42]. The nerve graft was found to be filled by axons which ended at the transition zone between the graft and the host spinal cord, but apparently, sporadic axons traveled further back into the spinal cord where they might have formed functional synaptic contacts.

Electrophysiological assessment of conduction in a PN graft is a great technical challenge. It is difficult to establish that the physiologic effects obtained are due directly to electrical activation of neuronal fibers within the graft and not to current spread or conduction along intact tracts when the SCI is incomplete. Some reports claim that PNGs inserted after a thoracic level (T8) complete SCI are capable of being electrically stimulated and of conducting action potentials, although with limited success [64]. However, the compound action potentials (CAPs) evoked by direct graft stimulation have a very short latency $(0.9 \mathrm{~ms})$. The short distance between the stimulating electrode and recording electrodes create a technical challenge, making it difficult to distinguish with certainty CAPs from the stimulation artifact.

There are instances where function is unquestionably linked to axonal regeneration within a PNG. Salame and Dum [37] demonstrated that axons regenerating within a PN bridging the brainstem and ipsilateral cervical spinal cord conduct action potentials and form functional synapses. Importantly, stimulation of the PN elicited electromygram responses in head and neck muscles that disappeared upon crushing the graft. Lee et al. (2002) found that in animals that received the combination of PNs and $\alpha \mathrm{FGF}$ into a mid-thoracic transection site had improved BBB scores and somatosensory- and motorevoked potentials (SSEP and MEP, respectively). Retransection of the spinal cord resulted in the loss of both SSEP and MEP, and the animals' BBB scores dropped to control group scores. Similarly, we demonstrated that combining PNG and ChABC promotes recovery of function following a cervical hemisection. Animals were better able to use their affected forepaw after the combined treatment than with PNG and vehicle, and this recovery was lost after the PNG was severed [26].

Perhaps the most successful model regarding electrophysiological evidence of functional recovery is nerve grafting and axonal regeneration of respiratory pathways. Gauthier and colleagues [59, 65, 66] have worked 
extensively on demonstrating functional axonal regrowth of central respiratory neurons. The proximal end of the peroneal nerve was acutely implanted in the medulla oblongata, in close proximity to respiratory cell bodies. Unitary discharges recorded from single fibers in the grafts confirmed that they were constituted of afferent and efferent respiratory pathways. Respiratory neurons that regenerated axons within the graft displayed spontaneous activity and had normal responses to respiratory activities (lung inflation/deflation, asphyxia, hyperventilation) [67]. If the distal end was left unapposed, spontaneous activity would stop after 3 months. Therefore, physiological function was maintained/regained in regenerating axons of respiratory neurons but this function was not preserved indefinitely in the absence of functional reconnection. When the distal end was apposed to the level of the phrenic nucleus, stimulation of the bridge elicited responses in the phrenic nerve that reflected a postsynaptic activation of the phrenic target [66]. These results indicate that, when provided with an appropriate denervated target, central respiratory neurons with regenerated axons along a nerve bridge can remain functional for a long period and can make precise and specific functional reconnections with appropriate target neurons.

There have been no reports of inappropriate functional activity (such as spasticity or pain) resulting from grafting of PN segments into the injured spinal cord. This does not preclude the possibility of aberrant synaptic contact by axons that emerge from the graft but at this time we have no evidence of undesired behavior following use of PNGs.

\section{Application of PNGs to a large animal SCI model}

Acute PNG experiments have been performed in cats $[36,68,69]$ and non human primates [70] to replicate findings in another species and in a larger animal model. Similar to what was observed in the early rat studies, axons grew into the grafts and they were myelinated by Schwann cells, but there was no evidence of growth back into the spinal cord [39]. Our recent work with cats sought to advance this early work by testing more current grafting strategies in a spinal cord that is closer in size to humans; the mean length of the feline spinal cord is $34 \mathrm{~cm}$ [71] versus $40-45 \mathrm{~cm}$ in humans [72]. Adult female cats received a lower cervical (C7) partial hemisection lesion with acute apposition of a segment of autologous tibial nerve to the lesion site [36]. Five weeks later a dorsal quadrant lesion cavity was created at $\mathrm{T} 1$, the lesion site was treated with ChABC 3 days later and the distal end of the PNG was apposed to the "fresh" injury site. Using morphological and electrophysiological techniques we were able to replicate many of the results obtained with rats. Grafts were full with several thousand myelinated axons. We observed BDA-labeled axons that had extended back into the cord. Axon conduction through the graft was established by stimulating the spinal cord rostral to the graft and recording from the graft surface. Furthermore, graft stimulation induced cFos in spinal cord neurons close to the distal apposition site (similar to our rat data), indicating synaptic activation through regenerated axons. We also were able to elicit electromyogram responses in the triceps muscle through antidromic activation of axons that had grown into the graft. Thus, using a cat SCI model, we are more able to measure physiological activity within the grafts opening the exciting possibilities for a more detailed analysis of any locomotor recovery and elucidating underlying electrophysiological mechanisms responsible for this recovery.

We have illustrated here that PN grafting is a promising repair strategy that has great potential to enhance functional recovery after SCI. It provides a permissive environment to support regeneration of injured axons and a physical substratum to direct them toward a specific target. This therapeutic approach is clinically relevant, showing successful regrowth in acute and chronic situations, with mild to severe SCI, at cervical or thoracic level and can functionally reestablish synaptic contact in respiratory, visual, motor and sensory systems. PNGs can unequivocally be part of a successful combinatory treatment strategy in SCI management, as continuing efforts are made to outline what complementary treatment will maximally promote structural and functional repair.

Acknowledgments: Portions of this work were supported by the National Institutes of Health Grant numbers NS026380 and NS055976, the Daniel Heumann Fund, the Paralyzed Veterans of America and the Craig H. Neilsen Foundation. Full conflict of interest disclosure is available in the electronic supplementary material for this article.

\section{REFERENCES}

1. Bray GM, Villegas-Pérez MP, Vidal-Sanz M, Aguayo AJ. The use of peripheral nerve grafts to enhance neuronal survival, promote growth and permit terminal reconnections in the central nervous system of adult rats. J Exp Biol 1987;132:5-19.

2. Aguayo AJ. Peripheral nerve transplantation techniques to study axonal regeneration from the CNS of adult mammals. In: Neural Grafting in the Mammalian CNS. Stenevi U, ed. Amsterdam, Netherlands: Elsevier Science Publishers, 1985:61-69.

3. David S, Aguayo AJ. Axonal elongation into peripheral nervous system "bridges" after central nervous system injury in adult rats. Science 1981;214:931-933.

4. Richardson PM, McGuinness UM, Aguayo AJ. Axons from CNS neurons regenerate into PNS grafts. Nature 1980;284:264-265.

5. Richardson PM, McGuinness UM, Aguayo AJ. Peripheral nerve autografts to the rat spinal cord: studies with axonal tracing methods. Brain Res 1982;237:147-162.

6. Richardson PM, Issa VM, Aguayo AJ. Regeneration of long spinal axons in the rat. J Neurocytol 1984;13:165-182.

7. Aguayo AJ, David S, Bray GM. Influences of the glial environment on the elongation of axons after injury: transplantation studies in adult rodents. J Exp Biol 1981;95:231-240. 
8. Ard MD, Bunge RP, Bunge MB. Comparison of the Schwann cell surface and Schwann cell extracellular matrix as promoters of neurite growth. J Neurocytol 1987;16:539-555.

9. Hall SM. Regeneration in cellular and acellular autografts in the peripheral nervous system. Neuropathol Appl Neurobiol 1986;12:27-46.

10. Stoll G, Muller HW. Nerve injury, axonal degeneration and neural regeneration: basic insights. Brain Pathol 1999;9:313-325.

11. Bamber NI, et al. Neurotrophins BDNF and NT-3 promote axonal re-entry into the distal host spinal cord through Schwann cellseeded mini-channels. Eur J Neurosci 2001;13:257-268.

12. Chau $\mathrm{CH}$, et al. Chondroitinase $\mathrm{ABC}$ enhances axonal regrowth through Schwann cell-seeded guidance channels after spinal cord injury. FASEB J 2004;18:194-196

13. Golden KL, et al. Transduced Schwann cells promote axon growth and myelination after spinal cord injury. Exp Neurol 2007;207:203-217.

14. Guest JD, et al. The ability of human Schwann cell grafts to promote regeneration in the transected nude rat spinal cord. Exp Neurol 1997;148:502-522.

15. Iannotti C, et al. Glial cell line-derived neurotrophic factor-enriched bridging transplants promote propriospinal axonal regeneration and enhance myelination after spinal cord injury. Exp Neurol 2003;183:379-393.

16. Menei $\mathrm{P}$, et al. Schwann cells genetically modified to secrete human BDNF promote enhanced axonal regrowth across transected adult rat spinal cord. Eur J Neurosci 1998;10:607-621.

17. Oudega $\mathrm{M}, \mathrm{Xu} \mathrm{XM}$. Schwann cell transplantation for repair of the adult spinal cord. J Neurotrauma 2006;23:453-467.

18. Paino CL, Bunge MB. Induction of axon growth into Schwann cell implants grafted into lesioned adult rat spinal cord. Exp Neurol 1991;114:254-257.

19. Xu XM, et al. Axonal regeneration into Schwann cell-seeded guidance channels grafted into transected adult rat spinal cord. J Comp Neurol 1995;351:145-160.

20. Xu XM, et al. A combination of BDNF and NT-3 promotes supraspinal axonal regeneration into Schwann cell grafts in adult rat thoracic spinal cord. Exp Neurol 1995;134:261-272.

21. Xu XM, et al. Bridging Schwann cell transplants promote axonal regeneration from both the rostral and caudal stumps of transected adult rat spinal cord. J Neurocytol 1997;26:1-16.

22. Turbes CC, Freeman LW. Peripheral nerve-spinal cord anastomosis for experimental cord transection. Neurology 1958;8:857-861.

23. Campos L, et al. Engineering novel spinal circuits to promote recovery after spinal injury. J Neurosci 2004;24:2090-2101.

24. Campos LW, et al. Regenerating motor bridge axons refine connections and synapse on lumbar motoneurons to bypass chronic spinal cord injury. J Comp Neurol 2008;506:838-850.

25. Friedman B, Aguayo AJ. Injured neurons in the olfactory bulb of the adult rat grow axons along grafts of peripheral nerve. J Neurosci 1985;5:1616-1625.

26. Houle JD, et al. Combining an autologous peripheral nervous system "bridge" and matrix modification by chondroitinase allows robust, functional regeneration beyond a hemisection lesion of the adult rat spinal cord. J Neurosci 2006;26:7405-7415.

27. Kerns JM, et al. The influence of predegeneration on regeneration through peripheral nerve grafts in the rat. Exp Neurol 1993;122:28 36.

28. Oudega M, Varon S, Hagg T. Regeneration of adult rat sensory axons into intraspinal nerve grafts: promoting effects of conditioning lesion and graft predegeneration. Exp Neurol 1994;129:194 206.

29. Bozkurt A, et al. In vitro cell alignment obtained with a Schwann cell enriched microstructured nerve guide with longitudinal guidance channels. Biomaterials 2009;30:169-179.

30. Ribeiro-Resende VT, et al. Strategies for inducing the formation of bands of Bungner in peripheral nerve regeneration. Biomaterials 2009;30:5251-5259.

31. Funakoshi H, et al. Differential expression of mRNAs for neurotrophins and their receptors after axotomy of the sciatic nerve. J Cell Biol 1993;123:455-465

32. Taniuchi M, et al. Expression of nerve growth factor receptors by Schwann cells of axotomized peripheral nerves: ultrastructural location, suppression by axonal contact, and binding properties. J Neurosci 1988;8:664-681.

33. Amin AA, JD Houle. The role of neurotrophic factors and their receptors in ascending and descending axon regeneration through intrapsinal peripheral nerve grafts (PNGs). 2010: Abstr Soc Neurosci 2010:588.28.

34. Hall SM. The effect of inhibiting Schwann cell mitosis on the reinnervation of acellular autografts in the peripheral nervous system of the mouse. Neuropathol Appl Neurobiol 1986;12:401-414.

35. Smith GV, Stevenson JA. Peripheral nerve grafts lacking viable Schwann cells fail to support central nervous system axonal regeneration. Exp Brain Res 1988;69:299-306.

36. Cote MP, et al. Peripheral nerve grafts after cervical spinal cord injury in adult cats. Exp Neurol 2010;225:173-82.

37. Salame CG, Dum RP. Central nervous system axonal regeneration into sciatic nerve grafts: physiological properties of the grafts and histologic findings in the neuraxis. Exp Neurol 1985;90:322-340.

38. Tom VJ, et al. Combining peripheral nerve grafts and chondroitinase promotes functional axonal regeneration in the chronically injured spinal cord. J Neurosci 2009;29:14881-14890.

39. Cheng H, Cao Y, Olson L. Spinal cord repair in adult paraplegic rats: partial restoration of hind limb function. Science 1996;273:510-513.

40. Lee YS, Hsiao I, Lin VW. Peripheral nerve grafts and aFGF restore partial hindlimb function in adult paraplegic rats. J Neurotrauma 2002;19:1203-1216.

41. Lee YS, et al. Improvement of gait patterns in step-trained, complete spinal cord-transected rats treated with a peripheral nerve graft and acidic fibroblast growth factor. Exp Neurol 2010;224 (2):429-437.

42. Nordblom J, et al. Peripheral nerve grafts in a spinal cord prosthesis result in regeneration and motor evoked potentials following spinal cord resection. Restor Neurol Neurosci 2009;27:285-295.

43. Houle JD, et al. Combining peripheral nerve grafting and matrix modulation to repair the injured rat spinal cord. J Vis Exp 2009;33. http://www.jove.com/details.stp?id=1324 doi:10.3791/1324.

44. Kobayashi NR, et al. BDNF and NT-4/5 prevent atrophy of rat rubrospinal neurons after cervical axotomy, stimulate GAP-43 and Talpha1-tubulin mRNA expression, and promote axonal regeneration. J Neurosci 1997; 17:9583-9595.

45. Storer PD, Dolbeare D, Houle JD. Treatment of chronically injured spinal cord with neurotrophic factors stimulates betaII-tubulin and GAP-43 expression in rubrospinal tract neurons. J Neurosci Res 2003;74:502-511.

46. Ye JH, Houle JD. Treatment of the chronically injured spinal cord with neurotrophic factors can promote axonal regeneration from supraspinal neurons. Exp Neurol 1997;143:70-81.

47. Malakhova OE, Davenport PW. cFos expression in the central nervous system elicited by phrenic nerve stimulation. J Appl Physiol 2001;90:1291-1298.

48. Pallini R, et al. Peripheral nerve autografts to the rat spinal cord: a study of the origin of regenerating fibres using fluorescent double labelling. Acta Neurochir Suppl (Wien) 1988;43:210213.

49. Gauthier P, Rasminsky M. Activity of medullary respiratory neurons regenerating axons into peripheral nerve grafts in the adult rat. Brain Res 1988;438:225-236.

50. Côté MP, et al. Activity-dependent increase in neurotrophic factors is associated with an enhanced modulation of spinal reflexes after SCI. J Neurotrauma 2011;28:299-309.

51. Houle JD. Demonstration of the potential for chronically injured neurons to regenerate axons into intraspinal peripheral nerve grafts. Exp Neurol 1991;113:1-9.

52. Sandrow HR, et al. Aspiration of a cervical spinal contusion injury in preparation for delayed peripheral nerve grafting does not impair forelimb behavior or axon regeneration. Exp Neurol 2008;210:489 500 .

53. Storer PD, Houle JD. Beta II-tubulin and GAP 43 mRNA expression in chronically injured neurons of the red nucleus after a second spinal cord injury. Exp Neurol 2003;183:537-547.

54. Tetzlaff W, et al. Response of facial and rubrospinal neurons to axotomy: changes in mRNA expression for cytoskeletal proteins and GAP-43. J Neurosci 1991;11:2528-2544. 
55. Shumsky JS, et al. Delayed transplantation of fibroblasts genetically modified to secrete BDNF and NT-3 into a spinal cord injury site is associated with limited recovery of function. Exp Neurol 2003;184:114-130.

56. Kwon BK, et al. Reaxotomy of chronically injured rubrospinal neurons results in only modest cell loss. Exp Neurol 2002;177:332-337.

57. Kwon BK, et al. Survival and regeneration of rubrospinal neurons 1 year after spinal cord injury. Proc Natl Acad Sci U S A 2002;99:3246-3251.

58. Coumans JV, et al. Axonal regeneration and functional recovery after complete spinal cord transection in rats by delayed treatment with transplants and neurotrophins. J Neurosci, 2001;21:9334-9344.

59. Decherchi P, Gauthier P. Regeneration of acutely and chronically injured descending respiratory pathways within post-traumatic nerve grafts. Neuroscience 2002;112:141-152.

60. Tobias CA, et al. Delayed grafting of BDNF and NT-3 producing fibroblasts into the injured spinal cord stimulates sprouting, partially rescues axotomized red nucleus neurons from loss and atrophy, and provides limited regeneration. Exp Neurol 2003;184:97-113.

61. Park KK, et al. Promoting axon regeneration in the adult CNS by modulation of the PTEN/mTOR pathway. Science 2008;322:963-966.

62. Carmel JB, et al. Chronic electrical stimulation of the intact corticospinal system after unilateral injury restores skilled locomotor control and promotes spinal axon outgrowth. J Neurosci 30:10918-10926.

63. Udina E, et al. Electrical stimulation of intact peripheral sensory axons in rats promotes outgrowth of their central projections. Exp Neurol 2008;210:238-247.
64. Pinzon A, et al. Conduction of impulses by axons regenerated in a Schwann cell graft in the transected adult rat thoracic spinal cord. J Neurosci Res 2001;64:533-541.

65. Gauthier P, Decherchi P. [Regrowth of central respiratory pathways in neural graft. From research tool on the axonal regeneration to a strategy of post-traumatic reparation]. C R Seances Soc Biol Fil 1997;191:695-716.

66. Gauthier P, et al. Functional reconnections established by central respiratory neurons regenerating axons into a nerve graft bridging the respiratory centers to the cervical spinal cord. J Neurosci Res 2002;70:65-81.

67. Lammari-Barreault N, Rega P, Gauthier P. Central respiratory neuronal activity after axonal regeneration within blind-ended peripheral nerve grafts: time course of recovery and loss of functional neurons. Exp Brain Res 1994;98:238-244.

68. Wrathall JR, et al. Reconstruction of the contused cat spinal cord by the delayed nerve graft technique and cultured peripheral nonneuronal cells. Acta Neuropathol 1982;57:59-69.

69. Sceats DJ Jr, et al. Regeneration in peripheral nerve grafts to the cat spinal cord. Brain Res 1986;362:149-156.

70. Levi AD, et al. Peripheral nerve grafts promoting central nervous system regeneration after spinal cord injury in the primate. J Neurosurg 2002;96(2 suppl):197-205.

71. Blinkov SM, II Glezer, The human brain in figures and tables; a quantitative handbook. 1968: Basic Book Co.

72. Perese DM, Fracasso JE. Anatomical considerations in surgery of the spinal cord: a study of vessels and measurements of the cord. J Neurosurg 1959;16:314-325. 